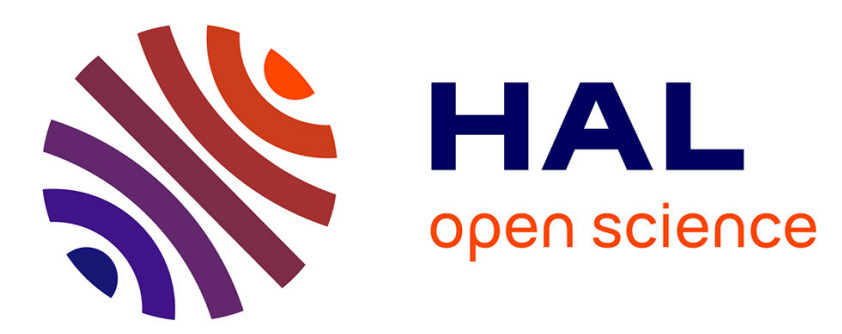

\title{
Granular Rayleigh-Taylor Instability: Experiments and Simulations
}

\author{
Jan Ludvig Vinningland, Øistein Johnsen, Eirik G. Flekkøy, Renaud \\ Toussaint, Knut Jørgen Måløy
}

\section{> To cite this version:}

Jan Ludvig Vinningland, Øistein Johnsen, Eirik G. Flekkøy, Renaud Toussaint, Knut Jørgen Måløy. Granular Rayleigh-Taylor Instability: Experiments and Simulations. Physical Review Letters, 2007, 99 (4), 10.1103/PhysRevLett.99.048001 . hal-01909759

\section{HAL Id: hal-01909759 https://hal.science/hal-01909759}

Submitted on 9 Nov 2018

HAL is a multi-disciplinary open access archive for the deposit and dissemination of scientific research documents, whether they are published or not. The documents may come from teaching and research institutions in France or abroad, or from public or private research centers.
L'archive ouverte pluridisciplinaire HAL, est destinée au dépôt et à la diffusion de documents scientifiques de niveau recherche, publiés ou non, émanant des établissements d'enseignement et de recherche français ou étrangers, des laboratoires publics ou privés. 


\title{
Granular Rayleigh-Taylor Instability: Experiments and Simulations
}

\author{
Jan Ludvig Vinningland, ${ }^{1, *}$ Øistein Johnsen, ${ }^{1}$ Eirik G. Flekkøy, ${ }^{1}$ Renaud Toussaint, ${ }^{2}$ and Knut Jørgen Måløy ${ }^{1}$ \\ ${ }^{1}$ Department of Physics, University of Oslo, P.O. Box 1048, N-0316 Oslo, Norway \\ ${ }^{2}$ Institut de Physique du Globe de Strasbourg, CNRS, Université Louis Pasteur, \\ 5 rue Descartes, 67084 Strasbourg Cedex, France \\ (Received 13 October 2006; published 24 July 2007)
}

\begin{abstract}
A granular instability driven by gravity is studied experimentally and numerically. The instability arises as grains fall in a closed Hele-Shaw cell where a layer of dense granular material is positioned above a layer of air. The initially flat front defined by the grains subsequently develops into a pattern of falling granular fingers separated by rising bubbles of air. A transient coarsening of the front is observed right from the start by a finger merging process. The coarsening is later stabilized by new fingers growing from the center of the rising bubbles. The structures are quantified by means of Fourier analysis and quantitative agreement between experiment and computation is shown. This analysis also reveals scale invariance of the flow structures under overall change of spatial scale.
\end{abstract}

DOI: 10.1103/PhysRevLett.99.048001

PACS numbers: 45.70.Qj, 47.11.-j, 47.20.Ma, 89.75.Da

Improved understanding of granular flows would be of essential importance to a range of industrial applications, to the study of geological pattern forming processes, and, in general, to the theoretical description of disordered media.

As grains become smaller the effect of the interstitial fluid becomes more important. The result is a combination of dry granular dynamics and the hydrodynamics of the fluid. These systems give rise to a variety of exotic and most often poorly understood phenomena such as fluidization [1] and bubble instabilities [2], quicksand and jet formation [3], and sandwich structures in systems where different particle types segregate [4]. While the study of dry granular media has been extensive over the past decades, the exploration of fluid-granular systems has been of more limited scope.

In this Letter we study a granular analog of the Rayleigh-Taylor instability [5] in the sense that an interface instability arises as a heavier phase (the grains) displaces a lighter phase (the air). The experimental setup consists of a closed Hele-Shaw cell that confines air and fine grains. When the cell is turned upside down we observe the evolution of an initially sharp front formed by the falling grains. This evolution has three stages: (1) An initial decompaction phase is followed by (2) the formation of vertical falling fingers (the dark filaments in Fig. 1) organizing into cusp-shaped structures that subsequently develop into (3) coarser finger-bubble structures. The last structures, seen in Fig. 1, represent a quasisteady state where two competing mechanisms produce a characteristic wavelength. The mechanism producing coarser scales originates as smaller bubbles lag behind bigger bubbles, giving rise to a finger merging process shaped like an inverted Y (see Fig. 1). This process resembles the coarsening seen in crystal growth [6]. The other mechanism, that produces finer scales and is active right from the start, is reminiscent of the tip splitting process seen in viscous fingering. It is manifested as thin filaments forming in the center of the rising bubbles.

Over the past few years a wide range of granular instabilities where various structures form along fluid-grain interfaces have been reported [2,7]. Notably, the patterns formed by grains falling in a highly viscous liquid were investigated experimentally and theoretically by Völtz et al. [8]. However, while the instability reported by Völtz shares its main qualitative characteristics with the classical Rayleigh-Taylor instability, i.e., a single dominating wavelength growing right from the start, our gas-grain instability grows through coarsening cusp structures.

The evolving structures further exhibit scale invariance under change of particle size, a feature which is supported both by observations and theoretical considerations. The simulations and experiments that are employed to shed light on the phenomenon at hand agree qualitatively, and
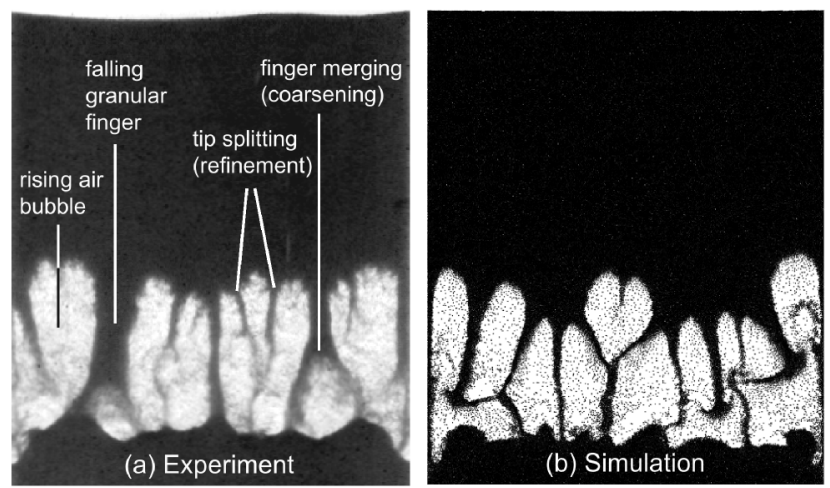

FIG. 1. (a) Experimental image and (b) numerical snapshot of a vertical Hele-Shaw cell where polystyrene beads (in black) of $140 \mu \mathrm{m}$ in diameter displace air (in white). The cells are $56 \mathrm{~mm}$ wide and were rotated $0.2 \mathrm{~s}$ ago. 
to a significant extent, quantitatively, even though the model neglects both granular friction and the spatial direction normal to the Hele-Shaw cell.

In the experiment a Hele-Shaw cell of inner dimensions $56 \mathrm{~mm} \times 86 \mathrm{~mm} \times 1 \mathrm{~mm}$ is partially filled with air and monodisperse beads (mass density $1.05 \mathrm{~g} / \mathrm{cm}^{3}$, diameter $140 \mu \mathrm{m})$ at atmospheric pressure. The cell is rotated manually in about $0.2 \mathrm{~s}$ from a lower to an upper vertical position to rapidly bring the layer of beads above the layer of air. Images of the evolving instability are recorded at a rate of 500 frames per second by a high speed digital camera with a resolution of $512 \times 512$ pixels; see Fig 1(a). A simultaneous numerical snapshot is given in Fig. 1(b).

The numerical model combines a continuum description of the air with a discrete description of the granular phase [9]. The effect of the granular phase on the air pressure is that of a deformable porous medium locally defined by the granular packing. The granular phase is modeled as discrete particles from which coarse grained solid fraction $\rho(x, y)$ and velocity fields $\mathbf{u}(x, y)$ are obtained by means of a linear smoothing function [9]. This function distributes the mass and velocity of a particle among its four neighboring grid nodes ( 2.5 grain diameters apart). The continuum gas phase is described solely by its pressure $P(x, y)$. The inertia of the gas, and hence its velocity field, is not considered. This is justified for small particle Reynolds numbers which is the case for our system.

The pressure is governed by the equation [9]

$$
\phi\left(\frac{\partial P}{\partial t}+\mathbf{u} \cdot \nabla P\right)=\boldsymbol{\nabla} \cdot\left(P \frac{\kappa(\phi)}{\mu} \boldsymbol{\nabla} P\right)-P \boldsymbol{\nabla} \cdot \mathbf{u},
$$

where $\phi=1-\rho$ is the porosity, $\kappa$ the permeability, $\mathbf{u}$ the granular velocity field, and $\mu$ the gas viscosity. This equation is derived from the continuity of air and grain mass, and Darcy's law with permeability $\kappa$. The Carman-Kozeny relation is assumed for the permeability, and the isothermal ideal gas law for the air.

The grains are governed by Newton's second law:

$$
m \frac{d \mathbf{v}}{d t}=m \mathbf{g}+\mathbf{F}_{I}-\frac{V \nabla P}{\rho},
$$

where $m, \mathbf{v}$, and $V$ are, respectively, the mass, velocity, and volume of the grain. Contact dynamics [10] is used to calculate the interparticle force $\mathbf{F}_{I}$ which keeps the grains from overlapping. The dynamics of the grains are simplified by neglecting particle-particle and particle-wall friction. A lower cutoff is imposed on the solid fraction because the Carman-Kozeny relation is not valid as the solid fraction drops below 0.25 [11]. This cutoff causes the permeability of the most dilute regions of the system to be slightly lower than the true permeability. The effect is a slight overestimation of the pressure forces acting on the grains in the dilute regions.

The spatiotemporal evolution of the air-grain interface in the experiment and simulation is presented in Figs. 2(b) and 2(e), respectively. For every horizontal position $x$ the interface height $y(x)$ is defined in the following way: Moving down from the top, $y(x)$ is the height where $\rho(x, y)$ drops below a given threshold value [see Figs. 2(a) and 2(d)]. Because of the large density contrasts in the system the interface position is rather insensitive to the exact value of this threshold. For the experimental data the threshold is set on the gray levels in the images.

The shape of the initial interfaces in Figs. 2(b) and 2(e) is quite different. The initial experimental interface has noise on all wavelengths, whereas the initial numerical interface is virtually flat with noise dominantly at smaller wavelengths. Perturbations introduced in the cell by the rotation and sudden stop disturb the initial experimental interface. However, as the instability evolves the discrepancy between experiment and simulation reduces and the later interfaces are in better agreement.

In order to give a more quantitative comparison of the interfaces, the discrete Fourier transform with a Hamming window is applied on every second interface in Figs. 2(b) and 2(e) to produce the power spectra presented in Figs. 2(c) and 2(f). The power spectra are colored as their corresponding interfaces, and the location of the maximum wave number for each power spectrum is indicated by a circle. While the maximum wave number of the numerical interfaces moves from high values to low values, the maximum wave number of the experimental interfaces hardly moves at all, most likely because the experiment does not evolve from an initially flat interface. However, the experimental and numerical power spectra converge to approximately the same form when normalized.

To study the coarsening of the observed structures quantitatively we perform an average over the solid fraction for the entire system: The discrete Fourier transform and the power spectrum of each horizontal line of $\rho(x, y)$ are calculated. The averaged power spectrum $\bar{S}(k)$ is then obtained by averaging over all these horizontal power spectra. An average wave number is defined as $\langle k\rangle=\sum_{k} \bar{S}(k) k / \sum_{k} \bar{S}(k)$, where $1 / k$ is the wavelength. Likewise, we define the squared standard deviation $\sigma_{k}^{2}=$ $\sum_{k} \bar{S}(k) k^{2} / \sum_{k} \bar{S}(k)-\langle k\rangle^{2}$. For the experimental data the image pixel values are used to estimate the solid fraction.

Figure 3 shows the temporal evolution of $\langle k\rangle$ and $\sigma_{k}$ (inset) for one simulation and two experiments. The numerical curve starts out with a significantly higher wave number than the experimental curves. However, the numerical data decrease monotonically until they coincide with the experimental data at about $0.2 \mathrm{~s}$, after which the simulation and experiments show a similar coarsening behavior. Fingers are not observed in the experiment until $0.06 \mathrm{~s}$ have elapsed. During this time the grains merely form a dilute sheet that appears homogeneous on the experimental images. This particular experimental initial state is caused by the sudden stop of the cell and is the most probable reason for the initial discrepancy between simulation and experiment in Fig. 3. The fluctuations of $\langle k\rangle$ and 


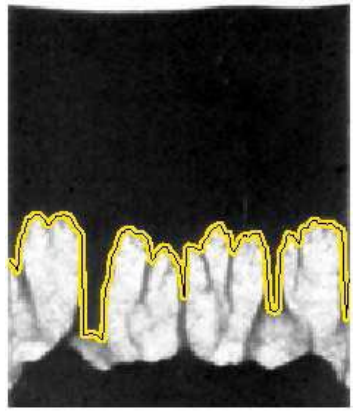

(a) Experiment

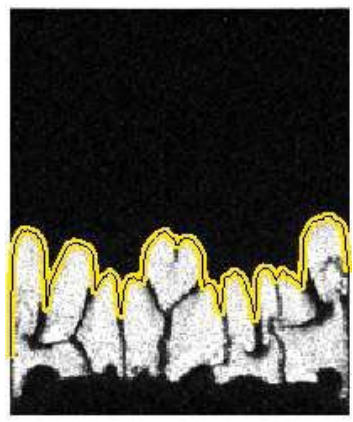

(d) Simulation

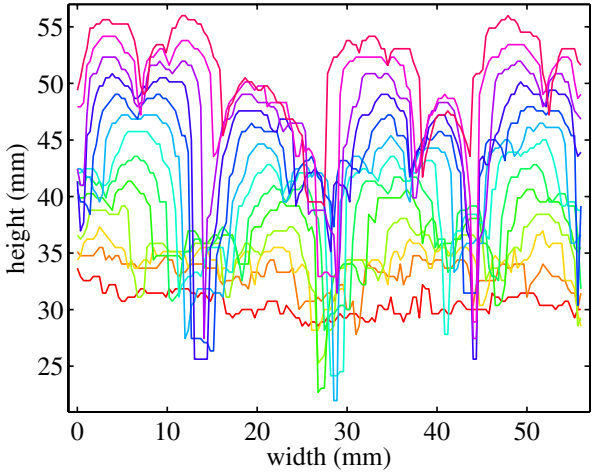

(b) Experiment, interface evolution

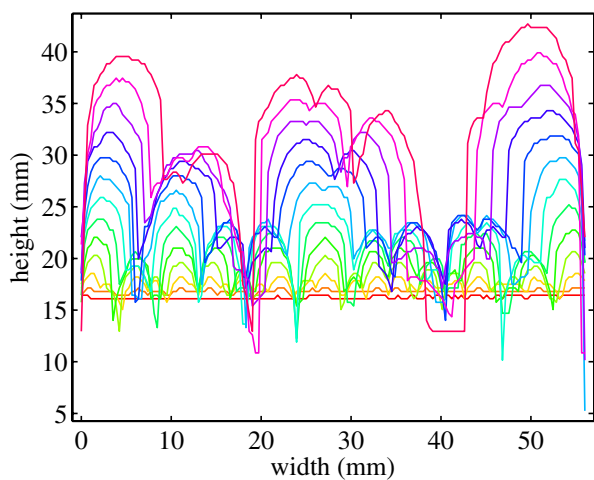

(e) Simulation, interface evolution

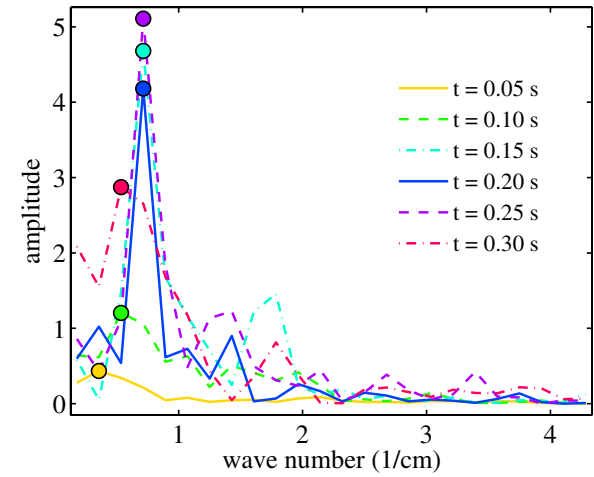

(c) Experiment, interface power spectrum

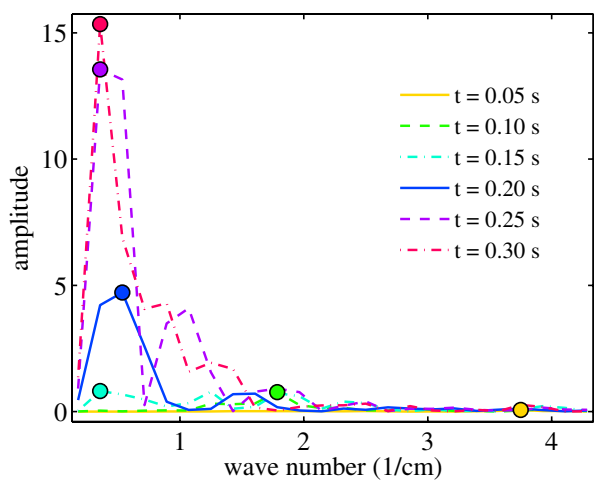

(f) Simulation, interface power spectrum

FIG. 2 (color online). (a),(d) Interfaces superposed on the snapshots $(t=0.20 \mathrm{~s}$ ) from Fig. 1. (b),(e) Spatiotemporal evolution of the air-grain interface from $t=0.00 \mathrm{~s}$ (bottom) to $t=0.29 \mathrm{~s}$ (top). (c),(f) Temporal evolution of the power spectrum of the interfaces. The circles indicate the location of the maximum wave number.

$\sigma_{k}$ are associated with the continuous nucleation and merging of fingers.

We further investigate the behavior of the system as the overall spatial scale is changed: Keeping all length ratios and the particle number fixed, the size of the system will scale according to the diameter $d$ of the grains. We measure the characteristic inverse length scale $\langle k\rangle$ as $d$ is changed and observe a scale invariance of the evolution. A series of seven simulations is performed where $d$ varies from 70 to $490 \mu \mathrm{m}$ in steps of $70 \mu \mathrm{m}$. The dimension of the numerical cell confining grains of $70 \mu \mathrm{m}$ in diameter is $28 \mathrm{~mm} \times$ $34 \mathrm{~mm}$. In these simulations we have introduced the larger density of glass, rather than polystyrene, in order to minimize the numerical artifacts associated with the solid fraction cutoff in the permeability. To compare, a series of experiments using polystyrene beads of $80,140,230$, and $570 \mu \mathrm{m}$ in diameter, confined in Hele-Shaw cells that scale proportionally with $d$ in all directions, is performed.

Data-collapse plots of the rescaled mean wave number $d\langle k\rangle$ are shown in Figs. 4(a) (simulation) and 4(b) (experiment). These plots indicate that the characteristic size of the structures is invariant when size is measured in units of $d$; the number of grains that spans the width of the bubbles is the same for a wide range of grain sizes.

Theoretically, the scale invariance of the product $d\langle k\rangle$ may be interpreted as follows: Compared with the other terms of Eqs. (1) and (2), the $m d \mathbf{v} / d t, F_{I}$, and $P \boldsymbol{\nabla} \cdot \mathbf{u}$ terms may be shown to be small [12]. For that reason, these equations exhibit an approximate invariance under system size scaling. If we take $\delta P$ to be the pressure deviation from the background pressure, express the velocity of grain $i$ as $\mathbf{v}_{i}=\delta \mathbf{v}_{i}+\mathbf{u}_{0}$ and the locally averaged granular velocity as $\mathbf{u}=\delta \mathbf{u}+\mathbf{u}_{0}$, where $\mathbf{u}_{0}$ is the constant sedimenta-

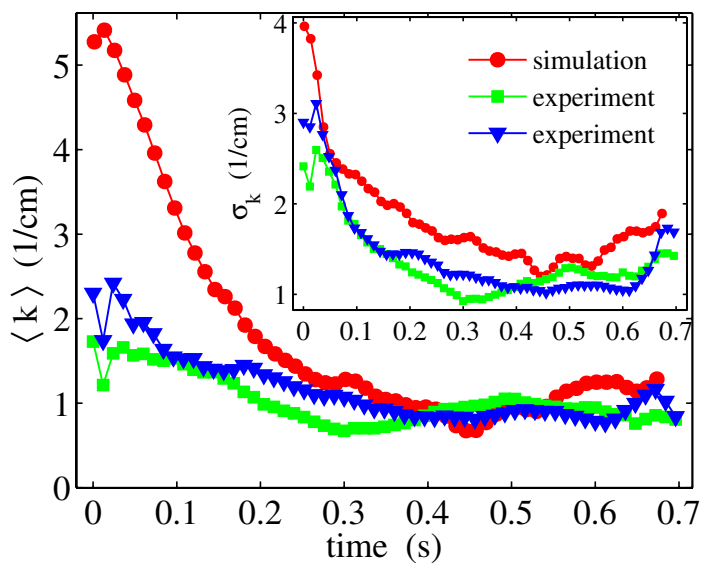

FIG. 3 (color online). Mean wave number $\langle k\rangle$ and standard deviation $\sigma_{k}$ (inset) for two experiments and one simulation, all using polystyrene beads of $140 \mu \mathrm{m}$ in diameter. 


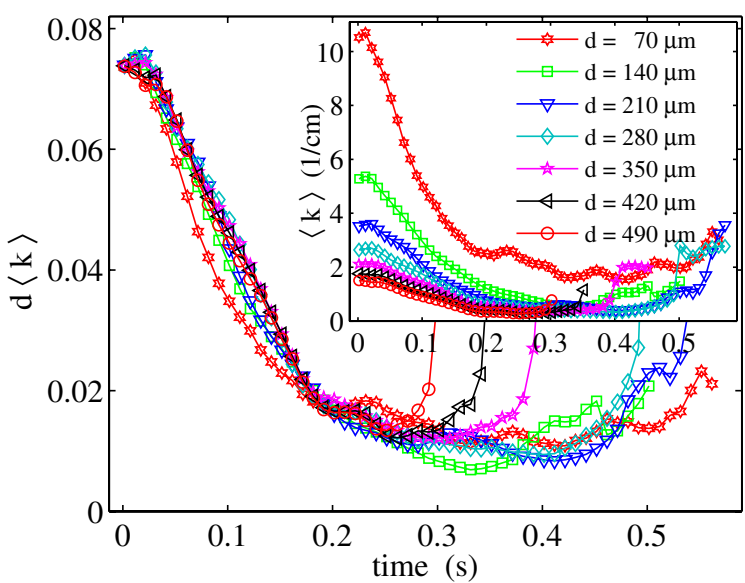

(a) Simulation

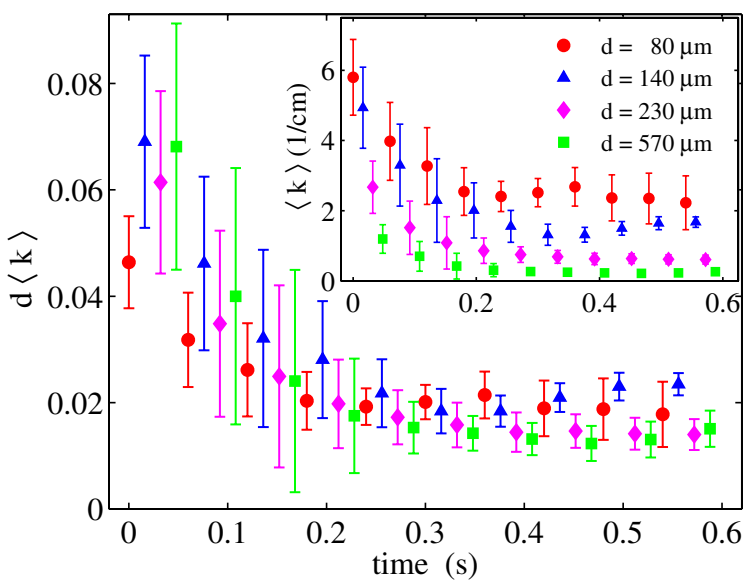

(b) Experiment

FIG. 4 (color online). Data-collapse plot of $d\langle k\rangle$ for a series of (a) simulations using glass beads and (b) experiments using polystyrene beads. The grain diameters $d$ are given in the legend box. The inset shows the evolution of $\langle k\rangle$.

tion velocity of a close packed system, this scaling may be expressed as $\mathbf{x} \rightarrow \lambda \mathbf{x}, \delta P \rightarrow \lambda \delta P, \mathbf{u}_{0} \rightarrow \lambda^{2} \mathbf{u}_{0}, \delta \mathbf{u} \rightarrow \lambda \delta \mathbf{u}$, and $\kappa \rightarrow \lambda^{2} \kappa$, where $\lambda$ is a scale factor. The structure formation of the system is governed by $\delta \mathbf{u}$ and, since this velocity scales the same way with $\lambda$ as the length scales themselves, the evolution of any structure measured in units of $d$ will be scale invariant. In particular, this is true for the structures measured by the length $1 /\langle k\rangle$, and so $d\langle k\rangle$ is scale invariant. However, the invariance deteriorates both when particle size is increased and when it is decreased. In the first case, the relative effect of granular inertia is increased, in the second, the relative effect of the $P \boldsymbol{\nabla} \cdot \mathbf{u}$ term is increased.

The convergence of the numerical data collapse in Fig. 4(a) is quite good. The deviation of the $70 \mu \mathrm{m}$ curve for small $t$ is probably explained by the increase in the relative importance of the $P \boldsymbol{\nabla} \cdot \mathbf{u}$ term. The divergences of the 350,420 , and $490 \mu \mathrm{m}$ curves for greater $t$ in the same plot arise because the bubbles in the coarser packings disappear before they reach the surface due to the increase of $\mathbf{u}_{0}$ with $\lambda^{2}$ [12]. The experimental data in Fig. 4(b) have a wider distribution but collapse satisfactorily given the standard deviation error bars. The experimental data are obtained by averaging over three experiments for each diameter $d$. The standard deviation is calculated over a time window of $0.3 \mathrm{~s}$. The accuracy of the experiments is at its lowest during the initial coarsening of the structures, but as the mean wave number stabilizes around $0.2 \mathrm{~s}$ the accuracy improves. Nevertheless, the data points are, with a few exceptions, within a distance of 1 standard deviation from one another. The loss of precision for small times is most likely caused by the inaccuracy involved with the manual rotation.

In conclusion, we have presented experimental and numerical results of a gravity-driven granular flow instability which is significantly different from its classical hydrodynamic analog. The simulations reproduce the characteristic shape and size of the experimentally observed structures and provide fine patterns in the early phase of the process that are not resolved experimentally. Data-collapse plots of the mean wave number $\langle k\rangle$ indicate that the flow and the resulting structures are invariant when measured on a scale proportional to the grain diameter $d$ for a range of diameters that spans from 70 to $570 \mu \mathrm{m}$.

*janlv@fys.uio.no

[1] Physics of Dry Granular Media, edited by H. J. Herrmann, J.-P. Hovi, and S. Luding (Kluwer Academic, Dordrecht, 1998); D. Gidaspow, Multiphase Flow and Fluidization (Academic, San Diego, 1994).

[2] D. Gendron, H. Troadec, K. J. Måløy, and E. G. Flekkøy, Phys. Rev. E 64, 021509 (2001); E. G. Flekkøy, S. McNamara, K. J. Måløy, and D. Gendron, Phys. Rev. Lett. 87, 134302 (2001).

[3] D. Lohse, R. Rauhé, R. Bergmann, and D. van der Meer, Nature (London) 432, 689 (2004).

[4] C. Zeilstra, M. A. van der Hoef, and J. A. M. Kuipers, Phys. Rev. E 74, 010302 (2006).

[5] Lord Rayleigh, Proc. London Math. Soc. s1-14, 170 (1883); G. Taylor, Proc. R. Soc. A 201, 192 (1950).

[6] J. S. Langer, Rev. Mod. Phys. 52, 1 (1980).

[7] C. Chevalier, M. B. Amar, D. Bonn, and A. Lindner, J. Fluid Mech. 552, 83 (2006); Ø. Johnsen, R. Toussaint, K. J. Måløy, and E. G. Flekkøy, Phys. Rev. E 74, 011301 (2006); F. Malloggi, J. Lanuza, B. Andreotti, and E. Clement, Europhys. Lett. 75, 825 (2006).

[8] C. Völtz, W. Pesch, and I. Rehberg, Phys. Rev. E 65, 011404 (2001).

[9] S. McNamara, E. G. Flekkøy, and K. J. Måløy, Phys. Rev. E 61, 4054 (2000); D.-V. Anghel, M. Strauss, S. McNamara, E. G. Flekkøy, and K. J. Måløy, Phys. Rev. E 74, 029906(E) (2006).

[10] F. Radjai, M. Jean, J.-J. Moreau, and S. Roux, Phys. Rev. Lett. 77, 274 (1996).

[11] A. A. Zick and G. M. Homsy, J. Fluid Mech. 115, 13 (1982).

[12] J. L. Vinningland, Ø. Johnsen, E. G. Flekkøy, R. Toussaint, and K. J. Måløy (unpublished). 